\title{
« Héros » et « héroïsmes » en Russie : notes pour une recherche
}

\section{Leonid Heller}

\section{(2) OpenEdition}

1 Journals

Édition électronique

URL : http://journals.openedition.org/edl/311

DOI : $10.4000 /$ edl.311

ISSN : 2296-5084

Éditeur

Université de Lausanne

\section{Édition imprimée}

Date de publication : 15 décembre 2009

Pagination : 71-80

ISBN : 978-2-940331-21-5

ISSN : 0014-2026

\section{Référence électronique}

Leonid Heller, « « Héros » et « héroïsmes » en Russie : notes pour une recherche », Études de lettres [En ligne], 4 | 2009, mis en ligne le 15 décembre 2012, consulté le 19 décembre 2020. URL : http://

journals.openedition.org/edl/311 ; DOI : https://doi.org/10.4000/edl.311 


\section{«HÉROS» ET «HÉROÏSMES» EN RUSSIE: NOTES POUR UNE RECHERCHE ${ }^{1}$}

L'article est consacré à l'épistémologie et à l'archéologie des notions "héros" et "héroïsme» en Russie, en comparaison avec la tradition héroïque européenne. Est discuté, entre autres, le phénomène aussi paradoxal que l'abolition de la frontière entre le collectif et l'individu, ainsi qu'entre le héros et l'homme ordinaire dans la vie quotidienne. En même temps, sont montrés les avatars inattendus et les conditions du fonctionnement discursif des notions de "héros", "grand homme», "athlète du travail», etc. dans les mondes russe et soviétique.

Le thème du héros est à la mode ${ }^{2}$. Des colloques et des ouvrages savants se multiplient qui interrogent la tradition héroïque européenne. En même temps, des chercheurs de plus en plus nombreux se penchent sur le rôle des héros dans d'autres traditions, depuis les épopées nationales de l'Amérique du Sud à l'émergence et à l'auto-affirmation de la culture afro-américaine. Par ailleurs, des héros et des super-héros submergent le cinéma, la télévision, les bandes dessinées, les jeux vidéos; leur présence dans la culture dite postmoderne est déjà devenue objet

I. L'article est écrit avec la collaboration de Korine Amacher (Genève).

2. Les remarques qui suivent sont extraites, avec quelques remaniements, de l'avantpropos du volume Le retour des héros. La reconstruction des mythologies nationales à l'heure du postcommunisme, à paraître en 2009 aux Editions Academia-Bruylant. Le colloque organisé par Korine Amacher (Genève) et le soussigné (Lausanne) a eu lieu à Genève en décembre 2007, sous les auspices de l'Institut européen de l'Université de Genève et de la Section slave de l'Université de Lausanne, dans le cadre du GEPECO, groupe suisse de recherches sur les pays de l'Europe centrale et orientale créé en 2003 et rattaché à l'Institut européen, cf. http://www.unige.ch/ieug/recherche/gepeco.html. 
d'expositions prestigieuses et de recherches académiques. On assiste à un courant d'idées d'une grande ampleur et on entend souvent la phrase de W. Benjamin: «Le héros est le vrai sujet de la modernité» ${ }^{3}$.

De nombreux travaux récents citent les formules de $\mathrm{M}$. Ozouf qui opposent le "héros" et le "grand homme». A la différence du dernier, le héros serait doté de pouvoirs surnaturels; il vivrait dans le temps de fulgurance et non, comme le grand homme, dans le temps lent et cumulatif; ses actions ne vaudraient rien sans le sacrifice ultime dans la mort ${ }^{4}$. On peut entrevoir là quelque parenté avec les théories de R. Girard ${ }^{5}$; le héros serait une sorte de bouc émissaire dont l'immolation assure la cohésion sociale.

Nous ne nous reconnaissons que partiellement dans ce cadre structurel imposé à la notion de "héros». Aussi, la tradition russe, telle du moins qu'elle nous apparait, et avec elle, paradoxalement, l'époque soviétique, valorisent-elles l'abnégation qui supplée au sacrifice; la mort n'est pas la seule voie vers l'héroïsme; et le héros ne se contente pas du rôle d'un étranger, d'un venu d'ailleurs qui traverse le paysage (on y reviendra plus loin).

Il semblerait que le russe n'a pas intégré le mot "héros» avant la deuxième moitié du XVII e siècle. Si l'on en croit les dictionnaires, le terme "héroïque" apparaît dans les textes anciens en rapport avec Homère pour dire 'épique'; l'«iros" fait son apparition dans la traduction difficilement datée de la Chronique de Jean Malalas ${ }^{6}$; c'est tout. Or la tradition orthodoxe, qui ignore le "héros", connaît bien le podvižnik et le podvig: ces mots sont censés correspondre aux agonistes et agon grecs et équivalent à peu près au "lutteur» et à la «lutte». Certes, il existait et il existe toujours le podvig militaire. Mais plus profondément, c'est de la lutte spirituelle dont il s'agissait, d'une lutte qui occupait une vie entière et ne s'éparpillait pas en actions spectaculaires. L'histoire d'Alexis homme de Dieu, le saint favori des Russes, qui passe quarante ans sous l'escalier de sa propre maison en mendiant et sans être reconnu, illustre bien la différence entre le podvižnik et les deux modèles occidentaux que

3. W. Benjamin, Charles Baudelaire, p. 108.

4. M. Ozouf, «Le Panthéon», p. 143.

5. Cf., par exemple, R. Girard, Le bouc émissaire.

6. R.I. Avanesov, Slovar' drevnerusskogo jazyka XI-XIV vv., t. IV, p. 166 ; S.G. Barxudarov, Slovar' russkogo jazyka XI-XVII vv., vol. 6, p. 249. 
nous avons évoqués plus haut, celui du "héros» comme celui du "grand homme».

Dans la littérature du XVIII siècle, les notions "héros" (iroj vs. geroj) et «hérö̈que» (gerojskij, geroičeskij) prennent de l'importance. La forme iroj, d'une forme et d'un emploi antiquisants, sera de plus en plus rare. La forme geroj hésitera entre plusieurs emplois et finira par tous les épouser. Le sens classique du mot entre dans les définitions de formes génériques (par exemple, "poème héroïque»: l'appellation de Télémachide (Tilemaxida, 1766) de V.K. Trediakovskij ${ }^{7}$; " héroïde» : récit consacré à la vie d'un héros), dans la versification (par exemple, le "vers héroïque" 8 ), la topique, l'imagerie. Dès 1730, A.D. Kantemir qualifie d'iroj le personnage principal d'une ouvre littéraire. Au milieu du XIX ${ }^{\mathrm{e}}$ siècle, le dictionnaire de V.I. Dal' aligne des synonymes du mot «héros» (vitjaz', bogatyr', voitel'...) pour noter, outre sa richesse sémantique, son ancrage dans la conscience linguistique russe; en même temps, il fait presque imperceptiblement glisser l'héroïsme de l'exploit individuel vers l'«action commune» [obšcij podvig] ${ }^{9}$.

Autour du héros, deux mouvements «idéologiques» se déploient en parallèle. D’un côté, la notion est sublimée, presque sacralisée. Certes, le romantisme y contribue, mais plus encore, l'inspiration religieuse et politique - impériale et nationale. Ce mouvement exploite la constellation sémantique et symbolique de podvig. Un héroïsme "officiel» se met en place; on peut en glaner les ingrédients dans le titre à rallonge de cette brochure datant de la guerre de Crimée: Les exploits hérö̈ques des défenseurs glorieux de Sébastopol qui revèlent toute la richesse de l'âme de l'homme russe, patient, tendrement attaché à la Patrie, téméraire au combat, dévoué à ses commandants, habité par l'amour du Tsar et une chaleureuse foi en Dieu [Gerojskie podvigi doblestnyx zaščitnikov Sevastopolja, $v$ kotoryx obnaruživaetsja vse bogatstvo duši russkogo čeloveka, ego terpenie, nežnaja privjazannost' $k$ rodine, molodeckaja udal' na službe iv dele, predannost' načal'nikam, liubov' $k$ carju i teplaja vera v Boga].

Pour les révolutionnaires populistes, narodniki, le mot "héros" a encore le goût du sacré. Mais il signifie autre chose que sacrifice de soi

7. V.K. Trediakovskij, Lirika, "Tilemaxida" i drugie sočinenija.

8. Soit l'«hexamètre» trediakovskien, soit le vers à treize syllabes avec une césure après la septième.

9. V.I. Dal', Tolkovyj slovar', vol. I, p. 859. 
et quelque chose de plus qu'abnégation, bien que ces attitudes puissent s'avérer nécessaires. Raxmetov, le prototype du héros révolutionnaire dans le célébrissime roman de N.G. Černyševskij, Que faire? [Čto delat'?] (1863), pratique à l'instar des yogis le contrôle de soi et la concentration, entraîne en permanence son esprit et son corps pour se tenir prêt à entrer à tout moment dans la lutte, à y donner toutes ses forces et à en supporter toutes les peines. Il est podvižnik: athlète de la foi et de la révolution.

Exaspéré par la fièvre des grandes actions, un écrivain très lu en son temps, I.N. Potapenko, intitule son livre-manifeste: Un non-héros [Ne-geroj] (1891).

C'est que le deuxième mouvement «idéologique» progresse, un mouvement de la décanonisation du héros, tantôt par l'intégration de ses référents à la vie quotidienne (l'héroïsme proche du podvig de l'artiste, du penseur, du philanthrope, même du paysan), tantôt par l'ironie - déjà à la fin du XVIII siècle, chez G.R. Deržavin:

Tak ne nado zvučnyx stroev,

Pereladim struny vnov';

Pet' otkažemsja geroev,

A načnem my pet' ljubov' ${ }^{\prime}$.

La désacralisation du héros procède par la mise en avant et une éventuelle confusion du héros-vedette et du héros-type, par l'insistance sur les liens du héros avec la mode et les mœurs du temps - on l'a vu avec M.Ju. Lermontov; bien que ces significations imitent les usages français, paradoxalement, elles attirent moins les Français que les Russes. Le mouvement culmine avec L.N. Tolstoj: non content de dénoncer l'imposture héroïque, il refuse au héros ce qui en constituait jusqu'ici sa principale raison d'être: l'efficacité de ses actions extraordinaires. Cet abaissement du «héros» va chez Tolstoj de concert avec celui du «grand homme». L'histoire se fait sans que l'un ou l'autre soit à même de l'influencer.

L'époque soviétique tente de faire fusionner les deux mouvements. A.V. Lunačarskij explique comment résoudre le problème du héros non démocratique qui attire l'attention des commentateurs d'aujourd'hui :

IO. "Trêve de sonorités sublimes, / Réaccordons nos instruments: / Refusons de chanter les héros - / Mettons-nous plutôt à chanter l'amour ", G.R. Deržavin, "K lire», p. 35 ; notre traduction. 
Le héros-leader joue un rôle exceptionnel, c'est un véritable cristal pour les volontés du collectif orientées dans la bonne direction, de ce collectif héroïque qui traverse les multitudes de souffrances. Or le culte du héros n'a pas lieu, le héros apparaît comme un organe naturel des masses. De telles masses ne peuvent manquer d'avoir de tels héros ${ }^{11}$.

Dès avant la révolution, la figure de l'Ouvrier combine les images de Héphaïstos, dieu des forges, du Titan récalcitrant Prométhée, de Spartacus, esclave révolté, et du saint martyr. Cette figure est destinée à entrer dans la culture soviétique, avec son corollaire négatif, la victime de l'exploitation capitaliste ${ }^{12}$. Le discours de la propagande aura vite intégré la notion de trudovoj podvig 'exploit du travail'. Dès 1921, le titre de Héros du travail est attribué à des travailleurs méritants et, en 1938, est instituée la plus haute distinction en temps de paix, Geroj socialističeskogo truda, le 'Héros du Travail socialiste'. Lorsqu'en 1935, Aleksej Staxanov est choisi pour personnifier l'ouvrier de choc stalinien, ce "héros» ayant accompli une action surhumaine, acquiert immédiatement la stature d'un véritable podvižnik. "Athlète du travail», il va pendant de longues années raconter son exploit et en déduire une méthode qui sera ensuite appliquée par des légions de «novateurs de la production" ${ }^{13}$.

Une héroïsation totale abolit la frontière entre le collectif et l'individu, rend l'action de la volonté humaine efficiente, fait abandonner ses errances au héros qui n'est plus jamais solitaire et sédentarise l'histoire, l'espace, la nature; les sacrifices sont toujours exigés, mais dans la durée, même si la médiatisation s'en empare souvent selon les modalités du vedettariat. En même temps, le héros soviétique est censé rester un homme ordinaire, placé dans le temps continu de tous les jours, mais qui doit faire de l'extraordinaire - «lorsque le Pays demande la présence de héros, tout un chacun chez nous devient un héros", comme le disent les paroles d'une chanson populaire ${ }^{14}-$, tout en servant de caution à

II. A.V. Lunačarskij, "O “Čapaeve”", p. 389.

I2. Cf., par exemple, V.A. Popov, Geroi i žertvy truda.

I3. A.G. Staxanov, Moj metod. Cf., par exemple, B.N. Agapov, Aleksej Grigor'evič Staxanov. Pour un regard décanonisant sur Staxanov et sur les stakhanovistes, cf. S.L. Talejsnik, " "Staxanovskaja operacija” i konec legendy".

I4. V.I. Lebedev-Kumač, "Marš veselyx rebjat». Sur l'emploi du héros dans l'éducation soviétique, cf. M. Heller, La machine et les rouages (chapitre "Education"); cf. également les analyses d'A. Zinoviev, par exemple, dans Le héros de notre jeunesse. 
des rites d'auto-remerciements: fêtes, distinctions, commémorations, publications. Nulle part ailleurs que dans la société soviétique n'est mieux mise en lumière la dialectique, traditionnelle dans la littérature moraliste russe, du "grand homme" et du "petit homme» (ou des "grandes âmes" et des "hommes gris", selon l'expression d'un chercheur ${ }^{15}$ ). Une série de livres portent ce titre: Geroi budnej, «les héros de la vie quotidienne» ${ }^{16}$. En apparence, l'opposition entre les "grands hommes", les "héros» et le "collectif» tend à disparaître; en réalité, divers "cultes de la personnalité» fonctionnant à des multiples niveaux réfutent l'équation lunačarskienne pour mettre en place une différence hiérarchique stricte entre les dirigeants, qui combinent à volonté leurs statuts de "grands hommes" et de "héros", et les autres membres de la société socialiste, dont le potentiel héroïque est exploitable sur commande.

La définition du héros est nécessairement multiple. Notre attention va vers tous les avatars héroïques si changeants. A l'époque soviétique, cela pouvait être le petit Pavlik Morozov, qui dénonça son père, ou Aleksandr Matrosov, qui bloqua avec sa poitrine le feu d'une mitrailleuse allemande ${ }^{17}$. Ou un grand homme, tel Pierre le Grand, ou un personnage inventé, tel Buratino d'A.N. Tolstoj, une variante de Pinocchio, soviétisée ou déstalinisée ${ }^{18}$. Staxanov, Morozov, Matrosov, Buratino sont autant des emblèmes que des produits du discours de leur époque. C'est ce fonctionnement discursif, avec ses conditions, ses tenants et aboutissants, que nous explorons: notre recherche continue.

Leonid HeLler

Université de Lausanne

I5. Ch. S. Kraszewski, The Romantic Hero and Contemporary Anti-hero.

16. Cf., par exemple, V. Gavrikov et al., Geroika budnej. Očerki o ljudjax socialističeskix polej; D. Rabinovič et al., Geroika budnej. Sbornik očerkov o ženščinax OKDVA; A. Tararuxin, Geroika budnej. Očerki i rasskazy o Moskovskoj krasnoznamennoj milicii; etc.

17. Cf., par exemple, Ju.I. Družnikov, Donosčik 001; P.T. Žurba, Geroj Sovetskogo Sojuza Aleksandr Matrosov.

I8. Cf. M.N. Lipoveckij, "Utopija svobodnoj marionetki, ili kak sdelan arxetip»; È.V. Nadtočij, «Roždenie i smert' total'nogo teatra». 


\section{BIBLIOGRAPHIE}

Agapov, Boris Nikolaevič, Aleksej Grigor'evič Staxanov, Moskva, Politizdat, 1938.

Avanesov, Ruben Ivanovič (otv.red.léd.), Slovar' drevnerusskogo jazyka XI-XIV vv. v 10 tomax, Moskva, Russkij jazyk, 1988-2000.

Barxudarov, Stepan Grigor'evič, Slovar' russkogo jazyka XI-XVII vv., vol. 6, Moskva, Nauka, 1979.

Benjamin, Walter, Charles Baudelaire: un poète lyrique à l'apogée du capitalisme [Charles Baudelaire: ein Lyriker im Zeitalter des Hochkapitalismus], tr. Jean Lacoste, Paris, Payot, 1979 (19381939).

ČernyšEvskij, Nikolaj Gavrilovič, Čto delat'?: iz rasskazov o novyx ljudjax, Moskva, Xudožestvennaja literatura, 1980 (1863).

DAL', Vladimir Ivanovič, Tolkovyj slovar' živogo velikorusskogo jazyka, Moskva, Izdatel'skaja gruppa "Progress »/«Univers», 1994, vol. I-IV (1903-1909).

Deržavin, Gavriil Romanovič, "K lire» (1797), in G.R. Deržavin, Anakreontičeskie pesni, Moskva, Nauka, 1986, p. 35.

Družnikov, Jurij Il'ič, Donosčik 001, ili voznesenie Pavlika Morozova, Moskva, Russkij put', 2006.

Gavrikov Vas[ilij] (Zılov, Lev Nikolaevič) et al., Geroika budnej. Očerki o ljudjax socialističeskix polej, Moskval Leningrad, Gosudarstvennoe izdatel'stvo sel'skoxozjajstvennoj i kolxozno-kooperativnoj literatury, 1931.

Gerojskie podvigi doblestnyx zašcitnikov Sevastopolja, v kotoryx obnaruživaetsja vse bogatstvo duši russkogo čeloveka, ego terpenie, 
nežnaja privjazannost' $k$ rodine, molodeckaja udal' na službe i v dele, predannost' načal'nikam, liubov' $k$ carju i teplaja vera $v$ Boga, Moskva, V Tipografii T.T. Volkova i Kº 1855.

Girard, René, Le Bouc émissaire, Paris, Grasset, 1982.

Heller, Michel (Geller, Mixail Jakovlevič), La Machine et les rouages. Histoire de la formation de l'homme soviétique, Paris, CalmannLévy, 1985.

Kraszewski, Charles S., The Romantic Hero and Contemporary Antihero in Polish and Czech Literature: Great Souls and Grey Men, Lewiston (N. Y.), The Edwin Mellen Press, 1998.

Lebedev-Kumač, Vasilij Ivanovič, «Marš veselyx rebjat», http://www. litera.ru/stixiya/authors/lebedevkumach/legko-na-serdce.html (1934).

LipoveckiJ, Mark Naumovič, "Utopija svobodnoj marionetki, ili kak sdelan arxetip (Perečityvaja "Zolotoj ključik" A.N. Tolstogo)", Novoe Literaturnoe Obozrenie, 60 (2003), p. 252-268.

LunaČArskij, Anatolij Vasil'evič, "O "Čapaeve”", in A.V. Lunačarskij,

Stat'i o literature, Moskva, GIHL, 1957, p. 388-391.

Nadtočij, Èduard Vadimovič, "Roždenie i smert' total'nogo teatra», Sinij divan, 2 (2003), p. 73-92.

Ozouf, Mona, "Le Panthéon. L'Ecole normale des morts", in Les lieux de mémoire, dir. Pierre Nora, Paris, Gallimard, 1984, t. 1, p. 139-166.

Popov, Vladimir Alekseevič (otv.red./éd.), Geroi i žertvy truda: izbrannye rasskazy (neobyčajnye istorii iz žizni trudovogo ljuda raznyx stran i narodov), Moskva/Leningrad, Zemlja i Fabrika, 1925.

Potapenko, Ignatij Nikolaevič, Ne-geroj, Moskva, Universitetskaja tipografija na Strastnom bul'vare, 1896.

Rabinovič, D. et al. (otv.red./éds), Geroika budnej. Sbornik očerkov o ženšcinax OKDVA (Osoboj Krasnoznamennoj Dal'nevostočnoj Krasnoj Armii), (s. l.) [Habarovsk], Dal'giz, 1936.

Staxanov, Aleksej Grigor'evič, Moj metod. Reč 14 nojabrja 1935 g., (s. 1.) [Moskva/Leningrad], Partizdat, 1935.

TalejSnik, Semen L'vovič, " "Staxanovskaja operacija” i konec legendy", http://www.andersval.nl

Tararuxin, A. (otv.red./éd.), Geroika budnej. Očerki i rasskazy o Moskovskoj krasnoznamennoj milicii, Moskva, Moskovskij rabočij, 1964. 
Trediakovskij, Vasilij Kirillovič, Lirika, "Tilemaxida» $i$ drugie sočinenija, Astraxan', Astraxanskij universitet, 2007.

Zinoviev, Alexandre (Zinov'ev, Aleksandr Aleksandrovič), Le héros de notre jeunesse: essai littéraire et sociologique sur le stalinisme, tr. Jacques Michaut, Paris/Lausanne, Julliard/L’Age d'homme, 1984 (1982).

Žurba, Pavel Terent'evič, Geroj Sovetskogo Sojuza Aleksandr Matrosov, Moskva, Voenizdat, 1949. 
